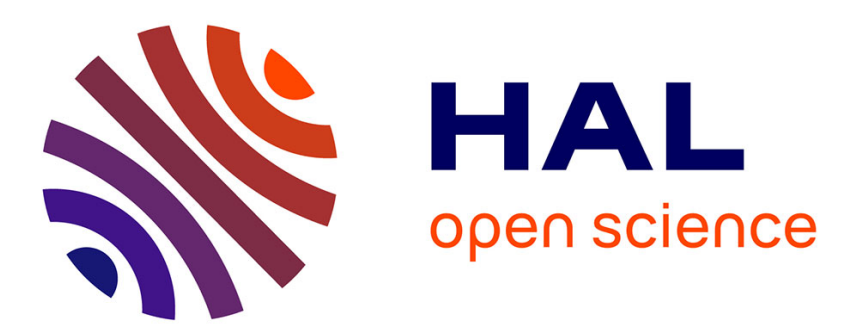

\title{
Relevance of visco-plastic theory in a multi-directional inhomogeneous granular flow
}

Pierre-Philippe Cortet, Daniel Bonamy, François Daviaud, Olivier Dauchot, Bérengère Dubrulle, Mathieu Renouf

\section{- To cite this version:}

Pierre-Philippe Cortet, Daniel Bonamy, François Daviaud, Olivier Dauchot, Bérengère Dubrulle, et al.. Relevance of visco-plastic theory in a multi-directional inhomogeneous granular flow. EPL Europhysics Letters, 2009, 88 (1), pp.14001. 10.1209/0295-5075/88/14001 . cea-01373382

HAL Id: cea-01373382

https://hal-cea.archives-ouvertes.fr/cea-01373382

Submitted on 28 Sep 2016

HAL is a multi-disciplinary open access archive for the deposit and dissemination of scientific research documents, whether they are published or not. The documents may come from teaching and research institutions in France or abroad, or from public or private research centers.
L'archive ouverte pluridisciplinaire HAL, est destinée au dépôt et à la diffusion de documents scientifiques de niveau recherche, publiés ou non, émanant des établissements d'enseignement et de recherche français ou étrangers, des laboratoires publics ou privés. 


\title{
Relevance of visco-plastic theory in a multi-directional inhomogeneous granular flow
}

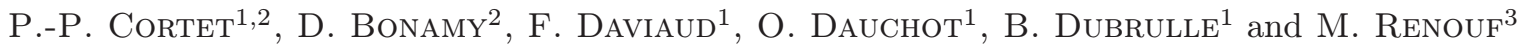 \\ ${ }^{1}$ CEA, IRAMIS, SPEC, CNRS URA 2464, Grp. Instabilités \& Turbulence - 91191 Gif-sur-Yvette, France, EU \\ ${ }^{2}$ CEA, IRAMIS, SPCSI, Grp. Complex Systems \& Fracture - 91191 Gif-sur-Yvette, France, EU \\ ${ }^{3}$ LaMCoS, CNRS UMR 5259, INSA Lyon - 18-20 rue des sciences, 69621 Villeurbanne, France, EU
}

received on 15 July 2009; accepted by L. F. Cugliandolo on 16 September 2009

published online 21 October 2009

PACS 45.70.-n - Granular systems

PACS 83.80.Fg - Granular solids

PACS $45.05 .+\mathrm{x}-$ General theory of classical mechanics of discrete systems

\begin{abstract}
We confront a recent visco-plastic description of dense granular flows (Jop P. et al., Nature, 441 (2006) 727) with multi-directional inhomogeneous steady flows observed in nonsmooth contact dynamics simulations of $2 \mathrm{D}$ half-filled rotating drums. Special attention is paid to check separately the two underlying fundamental statements into which the considered theory can be recast, namely i) a single relation between the invariants of stress and strain rate tensors and ii) the alignment between these tensors. Interestingly, the first prediction is fairly well verified over more than four decades of small strain rate, from the surface rapid flow to the quasi-static creep phase, where it is usually believed to fail because of jamming. On the other hand, the alignment between stress and strain rate tensors is shown to fail over the whole flow, what yields an apparent violation of the visco-plastic rheology when applied without care. In the quasi-static phase, the particularly large misalignment is conjectured to be related to transient dilatancy effects.
\end{abstract}

Copyright (c) EPLA, 2009

Despite granular flows ubiquity in nature and industry, their description still remains an open issue. Three regimes are usually distinguished: The rapid dilute flow - gaseous-like - regime, where grains interact through binary collisions, is described within the framework of the kinetic theory [1]; the slow flow — solid-like - regime, where only friction between grains is relevant, is described using soil mechanics [2]. In between, there is a denseflow - liquid-like - regime, where inertia becomes important, friction remaining however relevant. This last regime has been widely investigated experimentally and numerically (see [3] for a review) in various flow configurations and has been the focus of many theoretical works [4-8]. However, while these descriptions reproduce some of the features observed experimentally, a unified description is still missing. In particular, the possibility for a single and local constitutive law within the framework of continuum mechanics is still debated.

Progresses have been made recently in this context. Dimensional analysis $[3,9,10]$ was used to show that, in simple incompressible unidirectional uniformly sheared flows, bead diameter $d$, grain density $\rho$, global shear stress $\tau$, global pressure $P$ and global shear rate $\dot{\gamma}$ should be related as $\tau / P=\mu(I)$, where $\mu(I)$ is an effective friction coefficient, function of the so-called Inertial Number $I=$ $\dot{\gamma} d / \sqrt{P / \rho}$ expected to control locally the rheology of the material. Such relation has first been evidenced, numerically, in plane shear $[9,10]$ and, experimentally, in inclined plane [3] configurations. Local tensorial extension of this relation was recently proposed by Jop et al. [11] as a constitutive law for dense granular flows:

$$
\tau_{i j}=\mu(I) \frac{P}{\left|\dot{\gamma}^{d}\right|} \dot{\gamma}_{i j}^{d} \quad \text { with } \quad I=\frac{\left|\dot{\gamma}^{d}\right| d}{\sqrt{P / \rho}}
$$

where $\tau_{i j}=\sigma_{i j}+P \delta_{i j}$ denotes the deviatoric part of the stress tensor, $\dot{\gamma}_{i j}=1 / 2\left(\partial_{i} v_{j}+\partial_{j} v_{i}\right)$ is the strain rate tensor, $\left|\dot{\gamma}^{d}\right|=\sqrt{\frac{1}{2} \dot{\gamma}_{i j}^{d} \dot{\gamma}_{i j}^{d}}$ the norm of its deviatoric part $\dot{\gamma}_{i j}^{d}=\dot{\gamma}_{i j}-1 / 2 \dot{\gamma}_{k k} \delta_{i j}, P=-1 / 2 \sigma_{k k}$ the local pressure and $v_{i}$ the components of the velocity. In the compressible case, a consistent local rheology would imply in addition a univocal dependance $\nu=\nu(I)$ of the volume fraction on the inertial number [10]. Introducing eq. (1) in the momentum equation, Jop et al. succeeded to fit the surface velocity profile for a steady unidirectional flow down an inclined plane with walls. More recently, relation (1) was confronted with simulations of complex multi-directional 
non-steady inhomogeneous flow - the collapse of a cylinder of granular matter onto a horizontal plane [12] — and was shown to hold fairly well within the fast flowing region i.e. for $I$ ranging from $10^{-2}$ to 0.8 . In this last work, relation (1) has however been verified only for positions where stress and strain rate tensors had parallel principal directions, excluding $10 \%$ of data from the test. Therefore, despite these various experimental and numerical results, the relevance and limits of the visco-plastic relation (1) remain to be investigated in granular flows involving small values of inertial number $I$ - in which the stress and strain rate tensors are particularly prone to be non-aligned - as e.g. avalanches flows where large scale non-local effects are expected [13-16].

In the present letter, we confront this visco-plastic description of granular flows with steady surface flows observed in a 2D rotating drum, using extensive numerical simulations providing large statistics. The motivation for studying this specific flow is double since, still remaining in a "simple" $2 \mathrm{D}$ geometry, i) it provides a general highly multi-directional inhomogeneous situation and ii) it offers a wide range of inertial number: $10^{-6}<I<5 \times 10^{-2}$. In such a flow, we report an apparent failure of the constitutive relation (1) over the whole range of $I$, when applied in the "natural" frame of the flow. To overcome this failure, we highlight the fact that eq. (1) is actually composed of two independent results, namely a) a single relation between the invariants of stress and strain rate tensors and b) the alignment between these tensors, that constitute its two underlying fundamental hypotheses. The system of these two conditions actually recasts eq. (1) in a more fundamental formulation. Then, the norms of the stress and strain rate tensors are found to be univocally related through the invariant relation derived from eq. (1) over the whole range of $I$, meaning condition a) is verified over the whole flow. Finally, the failure of the tensorial relation (1) is shown to result from a misalignment between the stress and strain rate tensors - meaning condition b) fails - contrary to what is assumed in previous works. Possible origins of this misalignment are discussed: in the deep "solid" part of the flow, the total deformation experienced by the granular packing during its lifetime is less than one-bead diameter. This induces transient dilatancy effects - from a Lagrangian point of view - that could be at the origin of the persistence of the observed large misalignment.

The simulations have been performed using the NonSmooth Contact Dynamics method [17]. The scheme has been described in detail in [18] and is briefly recalled below. An immobile drum of diameter $D_{0}=45 \mathrm{~cm}$ is halffilled with 7183 rigid disks of density $\rho=2.7 \mathrm{~g} \mathrm{~cm}^{-3}$ and diameter uniformly distributed between 3 and $3.6 \mathrm{~mm}$. The weak polydispersity introduced in the packing prevents $2 \mathrm{D}$ ordering effects. The normal restitution coefficient between two disks (respectively, between disks and drum) is set to 0.46 (respectively, 0.46) and the friction coefficient to 0.4 (respectively, 0.95). Once the
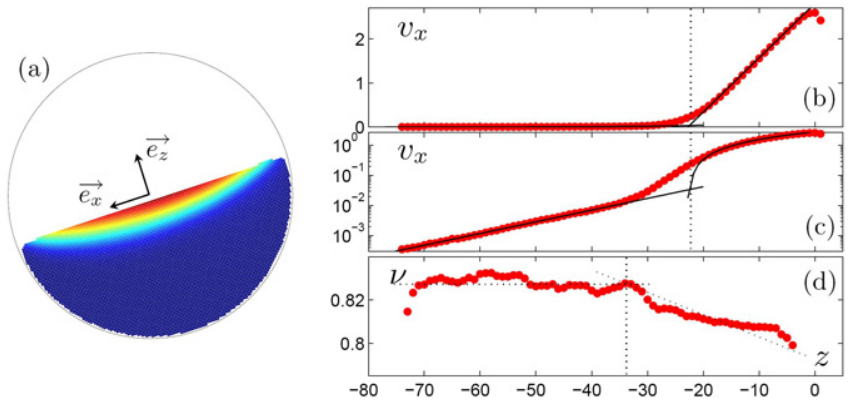

Fig. 1: (Colour on-line) (a)-(c) time and ensemble averaged streamwise velocity $v_{x}$ for a $\Omega=6 \mathrm{rpm}$ rotation velocity: (a) map with normalized colormap, (b) with linear and (c) logarithmic $z$-axis as a function of the depth $z$ at the center of the drum $(x=0)$. (d) Volume fraction $\nu$ as a function of the depth $z$ at the center of the drum $(x=0)$. Velocities are nondimensionalized by $\sqrt{g d}$, where $g$ is the gravity constant and $d$ the mean bead diameter. In (b) and (c), solid lines are the result of a linear and an exponential data fit.

initial packing is stabilized, a constant rotation velocity $\Omega$ ranging from 2 to $12 \mathrm{rpm}$ is imposed to the drum. After one round, a steady continuous surface flow has developed. Then, one starts to capture 400 snapshots equally distributed over one rotation of the drum. For each bead, one records the position $\vec{r}$ of its center of mass and its instantaneous velocity $\vec{c}$ over the time step $\delta t=6 \times 10^{-3} \mathrm{~s}$. For each rotation velocity, we performed 20 experiments starting from different initial packings. The reference frame $\Re$ is defined as the frame rotating at $\Omega$ with the drum that coïncides with the reference frame $\Re_{0}=\left(\overrightarrow{e_{x}}, \overrightarrow{e_{z}}\right)$ (cf. fig. 1), fixed in the laboratory, where $\overrightarrow{e_{x}}$ (respectively, $\overrightarrow{e_{z}}$ ) is parallel (respectively, perpendicular) to the mean flow free surface. The mean values of the various Eulerian fields (volume fraction, velocity and stress tensor) are obtained, at the grain scale (see [18]) and without any further coarse-graining, after both time and ensemble averaging over all the 400 frames of all the 20 experiments.

Steady surface flows in rotating drums provide a continuum of flow regimes from "quasi-static" to "liquid". Figures 1(b) and (c) present typical velocity profiles $v_{x}(x=0, z)$ measured in our numerical simulations "at the center" of the drum (i.e. along the $z$-aligned slice at $x=0$ ). Two phases can be identified: A flowing layer exhibiting a linear velocity profile and a "solid" phase experiencing creep motion where $v_{x}$ decays exponentially with $z$. Such velocity profile corresponds well to experimental measurements in rotating drum [3,19-21] as well as in heap geometry $[3,22]$. It is worth mentioning that the characteristic length $\xi$ associated with the exponential creep is found to be $\xi=12 \pm 2$ in our numerical drum over the range of explored rotating velocity. This is significantly larger than that reported by Crassous et al. [23] in heap geometry, $\xi=1 \pm 0.2$. This difference may emphasize the role played by the forcing on the selection of the velocity profiles within the bulk of the material. Indeed, the forcing 


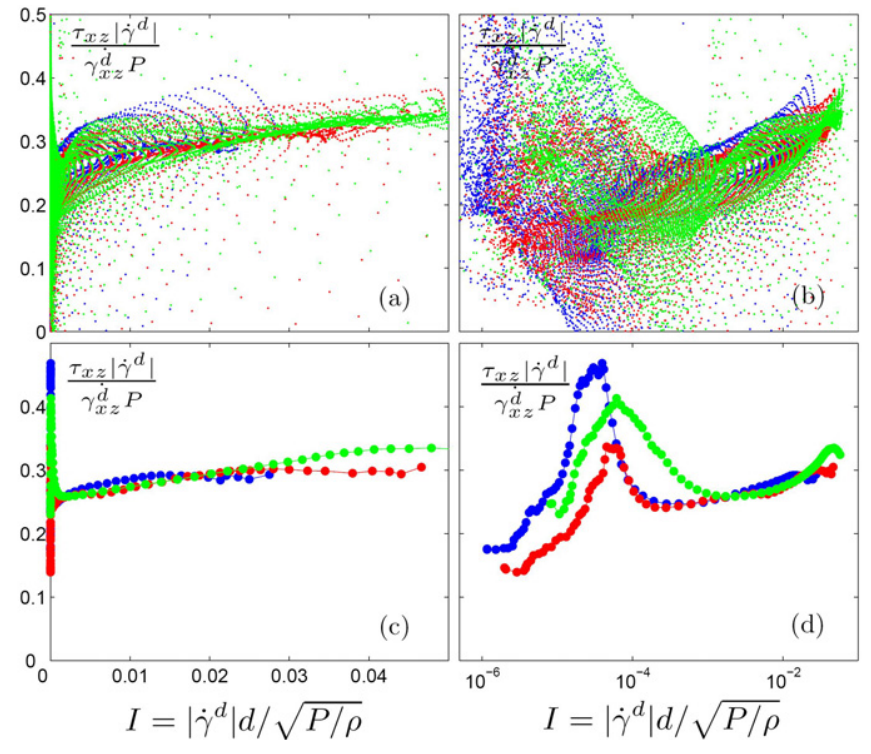

Fig. 2: (Colour on-line) Effective friction coefficient $\tau_{x z}\left|\dot{\gamma}^{d}\right| / \dot{\gamma}_{x z}^{\dot{d}} P$ as a function of the inertial number for time and ensemble averaged data, (a) and (b) over the whole 2D drum, (c) and (d) at the center of the drum $(x=0)$, with linear, (a) and (c), and logarithmic, (b) and (d), x-axis. Green/light gray points correspond to $\Omega=12 \mathrm{rpm}$, red/intermediate gray points to $\Omega=6 \mathrm{rpm}$, and blue/dark gray points to $\Omega=2 \mathrm{rpm}$.

in rotating drum and heap are very different since, from a Lagrangian viewpoint, the gravity is rotating in the first case while it remains frozen in the second. The volume fraction is found to be almost constant, around $0.827 \pm$ 0.05 , in the creeping layer while it decreases slightly in the flowing layer, down to 0.80 at $z=-4$ (cf. fig. 1(d)) as expected from dilatancy effects.

The full 2D fields of deviatoric stress $\tau_{i j}(x, z)$, deviatoric strain rate $\dot{\gamma}_{i j}^{d}(x, z)$ and pressure $P(x, z)$ are then computed to test the relevance of constitutive equation (1). Figures 2(a) and (b) show the variations of the ratio $\tau_{x z}\left|\dot{\gamma}^{d}\right| / \dot{\gamma}_{x z}^{d} P$ as a function of $I$ for time and ensemble averaged data corresponding to the whole $2 \mathrm{D}$ drum and for three different rotation velocities. If the rheology were correct, these data would collapse on the single master curve $\mu(I)$. This is clearly not the case as we observe an important dispersion of the data - even for the larger values of $I$. Additionally, as one can see in figs. 2(c) and (d), the collapse predicted by eq. (1) even does not work well (though better) when restricting data to the slice $x=0$ located at the center of the drum, where the flow is expected to be locally unidirectional $\left(/ /\right.$ to $\left.\vec{e}_{x}\right)$ and almost homogeneous $\left(\partial_{x} \simeq 0\right)$ as in the plane shear configuration. Moreover, the shape of these curves does not correspond to the logarithmic shape previously evidenced in $[3,10,11]$, especially, because of the unexpected bump of $\tau_{x z}\left|\dot{\gamma}^{d}\right| / \dot{\gamma}_{x z}^{d} P$ for $I$ ranging between $10^{-4}$ and $10^{-5}$.

The multi-directionality of the considered flow implies that no reference frame appears more natural than another to study tensors. Therefore, it is reasonable to recast tensorial equations into reference frame invariant relations. For granular flows, searching for a rheology consists mainly in relating the deviatoric stress tensor $\tau_{i j}$ to the deviatoric strain rate tensor $\dot{\gamma}_{i j}^{d}$. Since both of these tensors are symmetric with zero trace, both of them are fully characterized through one invariant only, e.g. their norm, and their principal directions. Accordingly, the rheology of eq. (1) can be recast in the following invariant way:
a) $\frac{|\tau|}{P}=\mu(I)$
b) $\tau_{i j}$ and $\dot{\gamma}_{i j}^{d}$ have identical principal directions,

where $|\tau|=\sqrt{\frac{1}{2} \tau_{i j} \tau_{i j}}$ is the deviatoric stress tensor norm. In unidirectional flows, such as those observed in plane shear geometry [10] or down to rough inclines [11], condition b) is naturally ensured by the flow symmetries so that verifying eq. (1) reduces to verifying point a) only. As we will see thereafter, this is no more the case for a multidirectional flow for which the alignment of the stress and strain rate tensors cannot be assumed $[24,25]$. The split of eq. (1) into the two independent conditions a) and b) recasts the rheology of Jop et al. into its two underlying fundamental assumptions which are completely independent. In the sequel, we will see that the invariant form of the visco-plastic rheology - condition a) - actually holds well over the whole rotating drum and the whole range of $I$ either condition b) holds or not. This reformulation of eq. (1) actually allows to test the visco-plastic theory for very small values of $I$ down to $10^{-5}$ where the condition on stress and strain rate tensor alignment is largely violated.

Let us first consider point a). In fig. 3 , we have plotted the invariant effective friction coefficient $|\tau| / P$ as a function of the inertial number for the same data as in fig. 2. We see that all the data appear to collapse quite well on a single master curve $\mu(I)$ independently of the rotation velocity $\Omega$. The shape of this master curve and the value of the corresponding friction coefficient are perfectly consistent with the ones already evidenced ( $\mu=$ $0.30 \pm 0.10$ for $I<0.1)$ in different granular unidirectional flows $[3,10,11]$. It is important to emphasize that this collapse is a non trivial result since we have plotted data for the whole 2D highly inhomogeneous fields. In figs. 3(c) and (d), we restrict data to the slice $x=0$ located at the center of the drum where the flow is expected to be almost "plane shear like". We see that the $|\tau| / P$ profiles for the three considered rotation velocities fall with a very good precision on the same master curve $\mu(I, x=0)$ for values of $I$ as small as $10^{-5}$. Despite this last remark, this collapse is a strong evidence for the possible relevance of the constitutive law of eq. (1) in steady inhomogeneous multi-directional flows. More importantly, it is the first time such frictional rheology seems to hold over such a low inertial number range $\left(10^{-5}<I<5 \times 10^{-2}\right)$ whereas it is 


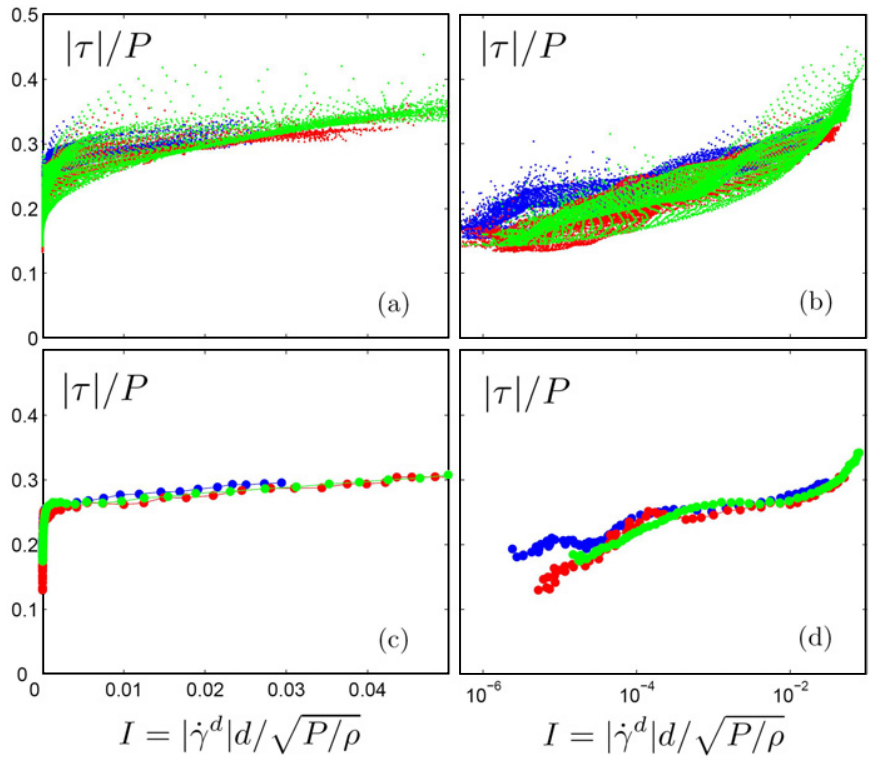

Fig. 3: (Colour on-line) Invariant effective friction coefficient $|\tau| / P$ as a function of the inertial number for time and ensemble averaged data, (a) and (b) over the whole 2D drum, (c) and (d) at the center of the drum $(x=0)$, with linear, (a) and (c), and logarithmic, (b) and (d), $x$-axis. Same color/gray level code as in fig. 2 .
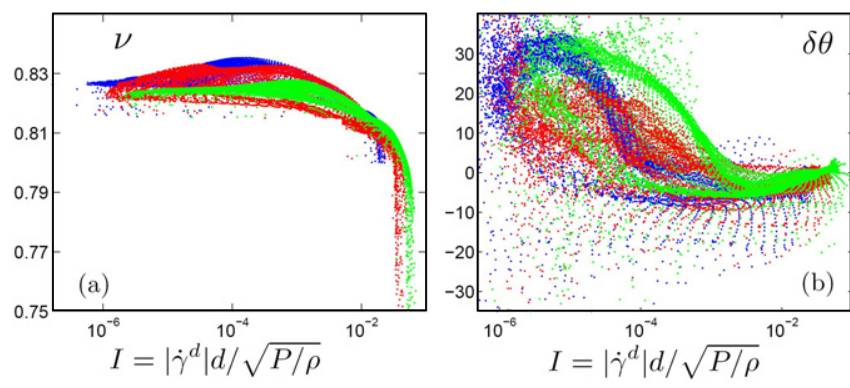

Fig. 4: (Colour on-line) (a) Volume fraction $\nu$ and (b) angle difference between principal directions of $\tau_{i j}$ and $\dot{\gamma}_{i j}^{d}$ as a function of the inertial number for time and ensemble averaged data corresponding to the whole 2D drum. Same color/gray level code as in fig. 2 .

often expected to fail because of the jamming transition for $I<10^{-3}$.

We already said that in compressive flows a local rheology would imply a single dependence of the volume fraction $\nu$ with the inertial number $I$. In fig. 4(a), we see that the data corresponding to the whole $2 \mathrm{D}$ flow collapse indeed quite well on a master curve $\nu=\nu(I)$ supporting partially the proposed rheological approach.

The second step is now to check point b) i.e. the collinearity between the deviatoric stress $\tau_{i j}$ and strain rate $\dot{\gamma}_{i j}^{d}$ tensors. Figure $4(\mathrm{~b})$ shows the angle difference $\delta \theta$ between the principal directions of $\tau_{i j}$ and $\dot{\gamma}_{i j}^{d}$ as a function of the inertial number. The collinearity is not verified since $\delta \theta$ ranges from $\pm 10^{\circ}$, for the larger inertial numbers $\left(5 \times 10^{-4}<I<5 \times 10^{-2}\right)$ corresponding
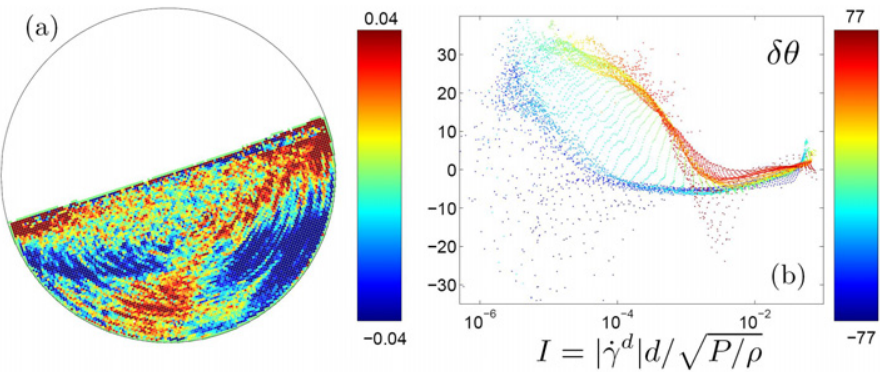

Fig. 5: (Colour on-line) (a) Map of the deviation $|\tau| / P-$ $|\tau| / P(x=0, z)$ of the effective friction coefficient $|\tau| / P$ from the one, $\mu(I, x=0)$, at the center of the drum for $\Omega=6 \mathrm{rpm}$. (b) Angle difference between principal directions of $\tau_{i j}$ and $\dot{\gamma}_{i j}^{d}$ as a function of the inertial number for $\Omega=12 \mathrm{rpm}$. In (b), data point colors are mapping the $x$-position in the drum.

to the "liquid" surface flow, up to $35^{\circ}$ in the deeper part of the flow, corresponding to smaller inertial numbers $\left(10^{-6}<I<5 \times 10^{-4}\right)$. Such increase in the dispersion of the tensors misalignment, as $I$ decreases, has also been reported in shear zones [24]. More importantly, there is no univocal relation ruled by the inertial number such as $\delta \theta=\delta \theta(I)$. Consequently, the fact the tensorial law (1) is not verified contrary to its invariant version, relation a), on the norms of the tensors holds is the direct consequence of the misalignment of the stress and strain rate tensors. Moreover, it shows, looking at the larger inertial numbers in figs. 2 and $4(\mathrm{~b})$, that a $10^{\circ}$ discrepancy between principal directions is already significant to make the visco-plastic tensorial rheology, in its non-invariant version of eq. (1), flatter.

In fig. 3 , the collapse of the data on a master curve $\mu(I)$ is not perfect and one may wonder if the dispersion around the "mean" friction coefficient $\mu(I, x=0)$ measured at the center of the drum is only due to a lack of statistics. Therefore, in fig. 5(a), we plot the map of the deviation of the effective friction coefficient from the one at the center of the drum. One can observe a spatial structuration with a quadripolar pattern. This spatial dependence of the profile $|\tau| / P=\mu(I, x)$ with the position $x$ may be the consequence of non-local effects reflecting the long range influence of the boundaries through force-chains, but remains however an unexplained feature. It is worth noticing that a pure geometrical dependence of $|\tau| / P$ has been reported in [25] where the interest of studying the present regime of mixed geometrical and shear rate dependence was highlighted. In parallel, in figs. 5(b) and $6(\mathrm{a})$, we see that the cloud of data $\delta \theta$ vs. $I$ shows as well a non-trivial spatial structure. There is however no evidence for any link between the spatial structures of the angle $\delta \theta$ and of the deviation around $\mu(I, x=0)$, the origins of which are still to be understood.

Previous studies $[24,25]$ have shown that the stress and the strain rate tensor principal directions can differ significantly due to transient dilatancy effects as long as the initial conditions of the packing have not been 

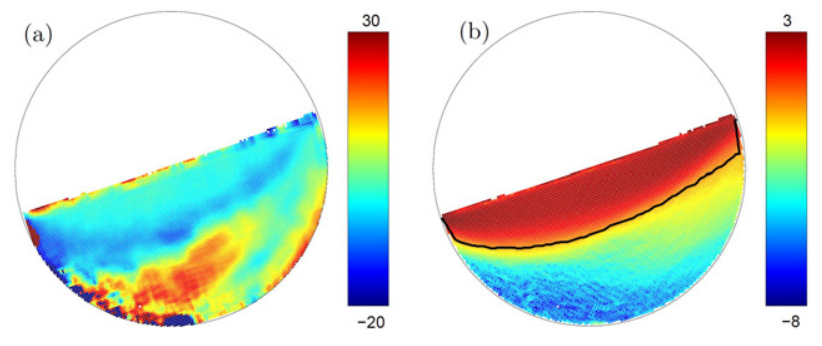

Fig. 6: (Colour on-line) (a) Map of the angle difference between principal directions of $\tau_{i j}$ and $\dot{\gamma}_{i j}^{d}$ for $\Omega=12 \mathrm{rpm}$. (b) Map of the logarithm of the norm of the strain rate tensor times the half drum rotation duration $\log \left|\dot{\gamma}^{d}\right| / 2 \Omega$ for $\Omega=12 \mathrm{rpm}$. We indicate with a black line the limit where this deformation $\delta \equiv\left|\dot{\gamma}^{d}\right| / 2 \Omega=1$.

forgotten, i.e. as long as the local deformation is less than $\sim 1$. Even if our flow is stationary, the transient effect reported above could be the explanation for the large misalignment observed in the deep part of the rotating drum (cf. fig. 6(a)). Indeed, in the "solid" part of the granular matter, a given "Lagrangian" packing is created at the bottom of the surface avalanche. Then, it has a short lifetime reduced to half a drum rotation period $1 / 2 \Omega$ corresponding to the time needed for him to reach the top of the avalanche where it is destructed. During this process, the deformation experienced by the packing is quite small: in fig. 6(b), we plot the map of the logarithm of the norm of the strain rate tensor times the half drum rotation duration $\log \left|\dot{\gamma}^{d}\right| / 2 \Omega$ as well as a black line delineating where the deformation $\delta=\left|\dot{\gamma}^{d}\right| / 2 \Omega$ equals unity. The "solid" granular matter under this line is experiencing a transient deformation since it is less than one. Finally, we see that this "transient" zone matches quite well the zone (cf. fig. 6(a)) where $|\delta \theta|$ is larger than $10^{\circ}$ making transient dilatancy effects a relevant explanation for the persistence of large alignment discrepancies. This observation does not imply that the spatial structure observed for the angle $\delta \theta$ is due to transient effects. On the contrary, this structure is created by the specific rotating-drum boundary conditions, the small deformations experienced by the matter explaining then the fact that this structure of $\delta \theta(x, z) \neq 0$ can live on.

Conclusion. - In this letter, we have confronted a recent visco-plastic theory for dense granular flow to numerical steady flows in rotating drums. The existence of a univocal relation between a scalar local friction coefficient - defined as the ratio between the norm of the deviatoric stress tensor to the pressure - and the socalled inertial number conjectured in the theory is in first approximation fairly well verified over the whole drum, in both the creeping "solid" phase and the avalanching phase at the free surface, for inertial numbers ranging from $10^{-5}$ to $5 \times 10^{-2}$. Small dilatancies evidenced at the flow surface are also found to be univocally related to the inertial number. On the other hand, the local alignment between deviatoric stress and strain rate tensors prescribed in the visco-plastic theory is found to fail significantly over the whole range of inertial number. This leads us to argue that the visco-plastic rheology proposed in [11] is extremely efficient to describe quasiunidirectional flows as those investigated in [9-11] but remains unapplicable, when applied in its non-invariant form (eq. (1)), to highly multi-directional flows such as those observed in rotating drum. On the other hand, it is particularly remarkable that the Jop et al. rheology, when considered in its invariant form, holds for very small values of $I$ down to $10^{-5}$ and that even if the stress and strain rate tensor misalignment is large. This result suggests in particular to reconsider the previous apparent failure of the visco-plastic rheology observed for small $I$ in other flow geometries $[10,25,26]$ which could have been a partially wrong conclusion. The origin of the observed misalignment between tensors is conjectured to be the consequence of transient dilatancy effects linked to the small deformation experienced by the "solid" part of the granular matter when considered from a Lagrangian point of view. Finally, a quadripolar spatial structuration of the dispersion around the perfect collapse of the data on the visco-plastic invariant rheology is tentatively attributed to non-local effects set by boundary conditions. In this context, stress distribution in immobile granular packings was found to be well described by Linear Elasticity [27]. It will then be interesting to see whether the introduction of an additional elastic field describing the stress distribution in an equivalent "immobile" packing can explain the observed discrepancy. Work in this direction is currently under progress.

This work was supported by the Triangle de la Physique and grant ANR TSF NT05-1-41492.

\section{REFERENCES}

[1] Savage S. B. and Jeffrey D. J., J. Fluid Mech., 110 (1981) 255.

[2] Nedderman R. M., Statics and Kinematics of Granular Materials (Cambridge University Press, Cambridge) 1992.

[3] Midi G. D. R., Eur. Phys. J. E, 14 (2004) 341.

[4] Savage S. B., J. Fluid Mech., 377 (1998) 1.

[5] Losert W. et al., Phys. Rev. Lett., 85 (2000) 1428.

[6] Mills P., Loggia D. and Texier M., Europhys. Lett., 45 (1999) 733.

[7] Aranson I. S. and Tsimring L. S., Phys. Rev. E, 65 (2002) 061303.

[8] Lemaitre A., Phys. Rev. Lett., 89 (2002) 064303.

[9] Iordanoff I. and Khonsari M. M., ASME J. Tribol., 14 (2004) 341.

[10] DA Cruz F. et al., Phys. Rev. E, 72 (2005) 021309.

[11] Jop P., Forterre Y. and Pouliquen O., Nature, 441 (2006) 727.

[12] Lacaze L. and Kerswell R. R., Phys. Rev. Lett., 102 (2009) 108305.

[13] Bonamy D. et al., Phys. Rev. Lett., 89 (2002) 034301. 
[14] Radjai F. and Roux S., Phys. Rev. Lett., 89 (2003) 064302.

[15] Pouliquen O., Phys. Rev. Lett., 93 (2004) 248001.

[16] Deboeuf S., Lajeunesse E., Dauchot O. and Andreotti B., Phys. Rev. Lett., 97 (2006) 158303.

[17] Moreau J.-J., Eur. J. Mech. A - Solids, 13 (1994) 93.

[18] Renouf M. et al., Phys. Fluids, 17 (2005) 103303.

[19] Rajchenbach J., Adv. Phys., 49 (2000) 229.

[20] Bonamy D., Daviaud F. and Laurent L., Phys. Fluids, 14 (2002) 1666.
[21] Courrech du Pont S. et al., Phys. Rev. Lett., 94 (2005) 048003.

[22] Komatsu T. S. et al., Phys. Rev. Lett., 86 (2001) 1757.

[23] Crassous J. et al., J. Stat. Mech. (2008) P03009.

[24] Ries A., Wolf D. E. and Unger T., Phys. Rev. E, 76 (2007) 051301.

[25] Depken M. et al., EPL, 78 (2007) 58001.

[26] Silbert L. E. et al., Phys. Rev. E, 64 (2001) 051302.

[27] Ovarlez G., Fond C. and Clement E., Phys. Rev. E, 67 (2003) 060302. 